\title{
Clinical Evaluation of Pharmacotherapy for Peptic Ulcer with Antipepsin Agents by Double Blind Technique - Multicenter Clinical Study -
}

\author{
Shoichi Yamagata and Akira Ishimori, Tohoku University School of \\ Medicine, Sendai \\ Hachiro Sato, Kagoshima University Medical School, Kagoshima \\ HaRuYa OKabe, Kyushu University School of Medicine, Fukuoka \\ KunI Ishinara, Tottori University Medical School, Yonago \\ Nagahiro Yugawa, Yugawa Hospital of Gastroenterology, Osaka \\ Masasuke Masuda, Kyoto Prefectural Medical College, Kyoto \\ Motonki Kishikawa, Faculty of Medicine, Nagoya City University, \\ Nagoya \\ Takao Sakita, National Cancer Center, Tokyo \\ Shinroku Ashizawa, Tokyo Medical College, Tokyo \\ Kenju Tsuneoka, Nippon Medical College, Tokyo \\ Isamu KaIto, Iwate Medical College, Morioka \\ Takeo WaDa, Sapporo Medical College, Sapporo \\ Masayoshi Namiki, Hokkaido University School of Medicine, Sapporo
}

Yamagata, S., Ishmori, A., Sato, H., OKabe, H., Ishrhara, K., Yugawa, N., Masuda, M., Kishrmawa, M., Sakita, T., Ashizawa, S., Tsuneoka, K., KatTo, I., WADA, T. and NaMTKI, M. Clinical Evaluation of Pharmacotherapy for Peptic Ulcer with Antipepsin Agents by Double Blind Technique. Tohoku J. exp. Med., 1973, 110 (4), 377-404_- In an attempt to examine the clinical effectiveness of pharmacotherapy for peptic ulcer through the standardized formula, clinical investigations were performed in a total of 255 cases of peptic ulcer at 13 medical institutes with the double blind technique. The antipepsin agents, namely degraded carrageenin was used as an active drug and aluminum sucrose sulfate as an active placebo, and lactose as an inactive placebo. The results indicated that both antipepsin agents are effective for the treatment of peptic ulcer without noticeable side effects. The therapeutic effect was more clearly shown on the following findings or symptoms, viz., $x$-ray and endoscopic findings, hypochodralgia, vomiting, epigastralgia, belching, meteorism, sense of distention, anorexia, retching, sense of gastric discomfort, abnormal fermentation, fecal occult blood reaction, tenderness, heartburn, constipation, and nausea. It was also disclosed that the global judgement of effectiveness had been made based chiefly on the x-ray and gastroscopic findings, and then on the outcome of such subjective symptoms as retching, vomiting, nausea, and anorexia. Problems involved in the clinical evaluation of pharmacotherapy for peptic ulcer by the double blind technique were also discussed.___ antipepsin agent; clinical 
evaluation of pharmacotherapy; double blind technique; peptic ulcer

A problem has recently been raised as to the method of evaluating the clinical effectiveness of pharmacotherapy for peptic ulcer (Piper 1967), and the double blind technique was introduced. Many methodological problems are, however, involved in the technique, and there is no standardized formula as yet.

To examine strictly the clinical effectiveness of pharmacotherapy for peptic ulcer with a standardized technique, the authors organized a committee consistsing of 13 medical institutes in Japan, and made clinical studies on antipepsin agents mentioned below for a period of about 1 year and 10 months. The results obtained are described in the present paper.

\section{Subjects and Methods}

\section{Subjects}

All patients with peptic ulcer encountered were employed as a subject for the study without any particular restriction on the sexuality, age, inpatient or outpatient, locality and size of ulcer, and primary or recurrent ulcer.

\section{Methods}

The double blind technique was employed. Since peptic alcer has a marked tendency toward spontaneous healing, the crossover system was not employed. And as experiments were to be carried out in many different institutes, the methods of administration of the agent and the methods of evaluation of the results obtained were discussed thoroughly beforehand, and the standardized same methods were employed in all institutes.

\section{1) Test agents}

To detect the clinical effectiveness o pharmacotherapy as purely and simply as possible, care was taken not to use other antiulcer agents than the test agents, so that the prominent clinical effectiveness can be expected. Antacids are not eligible apparently for this purpose and anticholinergic agents have also difficulties in the double blind technique which does not allow the individual adjustment of dose. For this reason antipepsin agents were chosen as the test agents in the present study. Namely degraded carrageenin was adopted as the active drug, aluminum sucrose sulfate (ulcerlmin); an antipepsin agent available in Japan as the active placebo, and lactose as the inactive placebo.

These active and inactive agents were all made into white granules, and packet was made to contain either $0.75 \mathrm{~g}$ of degraded carrageenin or aluminum sucrose sulfate, which was made to $1 \mathrm{~g}$ with lactose.

\section{2) Method of administration}

The above-mentioned three test agents were arranged at random according to the table of random numbers, and the bag thereof was given serial numbers for each medical institute, so that each institute would administer the test agents according to the serial numbers in the sequence of the visit of patients.

The administration of the test agents was standardized to be made 4 times a day at the time when the stomach was empty (Ishimori 1971) viz., at awakening early in the morning, before lunch, before dinner, and beforebedtime, and the administration was, in principle, continued for 12 weeks, unless ulcer was healed.

The combined use of other agents which might reduce the effects of the test agents or 
be identical or similar in the mechanism of action to the test agents was refrained. However, the anticholinergic agent could be injected only for a control of pain each time, and it was distinctly recorded.

3) Method of evaluation

The objective findings of ulcer lesion by endoscopy and $x$-ray examination were chiefly employed as a criteria of evaluating the effectiveness, and the fecal occult blood test and various subjective symptoms were also taken into consideration. These tests were, in principle, repeated at two-week intervals until it was endoscopically proved that the ulcer lesion-covering white coating had disappeared. In cases where no healing could be attained, however, the administration of the test agents was continued for 12 weeks, and then discontinued, when the experiments were terminted. The evaluation of the healing of duodenal ulcer was made by x-ray examination.

The change in ulcer lesion along with time was expressed in terms of the size of niche (length $\times$ width $\times$ depth) on $x$-ray film, and in terms of the ratio of the area of ulcer lesion before and after the administration of the test agents in the standardized endoscopic observations which were made as accurately as possible.

Such subjective symptoms were checked as epigastralgia, hypochondralgia, tenderness, nausea, heartburn, belching, retching, vomiting, discomfort in the gastric region, sense of distention, anorexia, dyspepsia, abnormal fermentation, meteorism, constipation, and diarrhea. The intensities of these symptoms were standardized to be expressed with $\mathrm{Ht}$ for such an intensity that the symptoms had to bə treated with periodic recipes for a considerably long period, \# treated with tentative recipes, + needed no particular treatment, and - for no symptoms.

Side-effects were checked throughout the experimental periods, and various clinical tests e.g., liver function test: ZTT, TTT, alkaline phosphatase activity, GOT and GPT activities, serum protin: serum total protein level and $A / G$ ratio, serum lipid level: triglyceride, phospholipid and total cholesterol, concentration of serum electrolytes such as $\mathrm{Na}$, $\mathrm{K}$ and $\mathrm{Cl}$, red blood cell count, hemoglobin level, white blood cell count, bleeding time, blood coagulation time, and further, wherever possible, thrombotest and prothrombin time were made at least before, and 4 weeks after the beginning of the treatment.

The evaluation of effectiveness of the test agents was made by the 5-step assessment method, viz., in 5 steps of "markedly effective", "effective", "relatively effective", "no change", and "exacerbated", based on all findings mentioned above and also on the free assessment by the physicians in charge.

The calculation of the results was made with electronic computer, and the numerals with asters in the figures and tables stand as follows: the single-asterisked $(*)$ ones stand for a significant difference at a $5 \%$ level of significance, the double-asterisked $\left(^{* *}\right)$ ones for a difference at a $1 \%$ level of significance, and the triple-asterisked $\left({ }^{* * *}\right)$ ones for a difference at a $0.5 \%$ level of significance.

\section{Results}

A total of 255 cases, in which 26 cases drop-out, were studied in 13 medical institutes. The rates of dropout were only $10.2 \%$, being below the usual drop-out rates of $12-13 \%$ for the experiments of this kind. This indicates that the double blind technique could be excused in a nearly ideal way.

The cases treated with the test agents are summarized in Table 1 in detail, showing the drop-outs and the subjects for analysis. The subjects for analysis comprised 70 cases given degraded carrageenin, 81 cases given aluminum sucrose sulfate, and 78 cases given lactose. Although there were some differences in number of cases among the three groups, the test for equality was: $\mathrm{X}_{\mathbf{0}}^{2}=0.8472<1.38629$ 
TABLE 1. Construction of cases reported

\begin{tabular}{|c|c|c|c|c|c|}
\hline & & $\begin{array}{c}\text { A. } \\
\text { Degraded } \\
\text { carrageenin } \\
\text { group }\end{array}$ & $\begin{array}{l}\text { *B. } \\
\text { Aluminum } \\
\text { sucrose } \\
\text { sulfate } \\
\text { group }\end{array}$ & $\begin{array}{l}\mathrm{P} . \\
\text { Lactose } \\
\text { group }\end{array}$ & Total \\
\hline & Number of cases reported & 82 & 89 & 84 & 255 \\
\hline & Drop-out & 12 & 8 & 6 & 26 \\
\hline \multirow{14}{*}{ 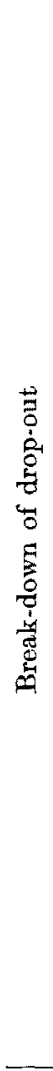 } & $\begin{array}{l}\text { Diagnosis established as gastric } \\
\text { cancer after } 10 \text { weeks }\end{array}$ & 1 & & , & 1 \\
\hline & $\begin{array}{l}\text { Subjected to operation after } \\
8 \text { weeks }\end{array}$ & 1 & & 1 & 2 \\
\hline & Discontinued due to exanthema & & 1 & & 1 \\
\hline & $\begin{array}{l}\text { Discontinued due to continuous } \\
\text { abdominal pain (after } 2 \\
\text { weeks) }\end{array}$ & 1 & 1 & & 1 \\
\hline & $\begin{array}{l}\text { Discontinued due to severe } \\
\text { subjective symptom (after } \\
3 \text { weeks) }\end{array}$ & 1 & & & 1 \\
\hline & Ditto (after 4 weeks) & 3 & 1 & & 4 \\
\hline & Ditto (after 6 weeks) & & & 1 & 1 \\
\hline & $\begin{array}{l}\text { Transferred to another hospital } \\
\text { due to myoma of the uterus }\end{array}$ & & 1 & & 1 \\
\hline & $\begin{array}{l}\text { Discontinued to visit hospital } \\
\text { after I week }\end{array}$ & 1 & 3 & & 4 \\
\hline & Ditto after 2 weeks & & & 1 & 1 \\
\hline & $"$ & 2 & & 1 & 3 \\
\hline & $"$ & & 1 & 1 & 2 \\
\hline & 10 & 1 & 1 & 1 & 3 \\
\hline & Mis-recognition of niche & 1 & & & \\
\hline & Number of cases for analysis & 70 & 81 & 78 & 229 \\
\hline
\end{tabular}

Rate of drop-out: $26 / 255=10.2 \%$

$=\mathrm{X}_{2}{ }^{2}(0.50)$, viz., there was no significant difference at a $5 \%$ level of significance, and this could be considered to represent the effect of random arrangement.

\section{1) Analysis of the global judgement of effectiveness}

In Table 2 are shown the results of the global judgement of effectiveness of the test agents made by the physicians in charge. The three medicated groups were independent for each oter, and each group comprised a sufficiently large number of 
TABLE 2. Global judgement made by doctors in charge, and relation between three groups tested by Kruskal-Wallis Test (H-Test)

\begin{tabular}{|c|c|c|c|c|c|c|c|}
\hline $\mathrm{k}$ (group) & $\begin{array}{l}\text { Marked- } \\
\text { ly ef- } \\
\text { fective }\end{array}$ & Effective & $\begin{array}{l}\text { Rela- } \\
\text { tively } \\
\text { effective }\end{array}$ & $\begin{array}{c}\text { No } \\
\text { change }\end{array}$ & $\begin{array}{c}\text { Exacer- } \\
\text { bated }\end{array}$ & $\begin{array}{l}\text { Total } \\
\text { (nj) }\end{array}$ & $\begin{array}{l}\text { Total of } \\
\text { orders of each } \\
\text { group }(R j)\end{array}$ \\
\hline $\begin{array}{l}\text { Degraded carr- } \\
\text { ageenin group }\end{array}$ & 30 & 20 & 18 & 2 & & 70 & $7,243(103.5)$ \\
\hline $\begin{array}{l}\text { Aluminum } \\
\text { sucrose sulfate } \\
\text { group }\end{array}$ & 29 & 30 & 16 & 5 & 1 & 81 & $8,895.5(109.8)$ \\
\hline Lactose group & 24 & 19 & 16 & 16 & 3 & 78 & $10,196.5(130.7)$ \\
\hline $\begin{array}{l}\text { Total cases } \\
\text { (cumulative } \\
\text { total) }\end{array}$ & 83 & $\begin{array}{c}69 \\
(152)\end{array}$ & $\begin{array}{c}50 \\
(202)\end{array}$ & $\begin{array}{c}23 \\
(225)\end{array}$ & $\begin{array}{r}4 \\
(229)\end{array}$ & 229 & 26,335 \\
\hline (Average order) & $(42.0)$ & $(118.0)$ & $(177.5)$ & $(214.0)$ & $(227.5)$ & & \\
\hline
\end{tabular}

$$
\mathrm{x}_{0}^{2}=7.6714 *>7.3778=\mathrm{x}_{2}^{2}(.025)
$$

Result of H-test: At $\mathrm{p}<0.025$, significant difference in drug was found between the three groups.

cases ( $\mathrm{nj}>20$ ), with a lattice below grade 5. For this reason, the $X^{2}$ test could not be applied, and the Kruskal-Wallis' test, (H-test) which is higher in the power of test than the $\mathrm{X}^{2}$ test based on mere nominal grade was employed. It gave $\mathrm{X}_{\mathbf{0}}{ }^{2}$ $=7.6714^{*}>7.3778=\mathrm{X}_{2}{ }^{2}(0.025)$, viz., significant differences were found to exist in the results of the global judgement of effectiveness among the three groupsat a $2.5 \%$ level of significance.

In order to examine a difference in therapeutic effects on the 3 combinations of any two of the 3 groups, Mann-Whiteney's test (U-test) was employed to compare the findings between two groups. First of all, the comparison of the degraded carrageenin group with the aluminum sucrose sulfate group revealed no significant difference at a $5 \%$ level of significance with $\mathrm{P}=0.5300$ (two-tailed) and $\mathrm{P}=0.2650$ (one-tailed) as seen in Table 3 (1). Thus no difference in effectiveness was found between degraded carrageenin and aluminum sucrose sulfate based on the results of the global judgement. On the other hand, the comparison of the degraded carrageenin group with the inactive placebo group gave $\mathrm{P}^{* *}=0.009478$ (two-tailed) and $\mathrm{P}^{* * *}=0.004739$ (one-tailed) as shown in Table 3 (2), and that of the aluminum sucrose sulfate group with the lactose group gave $\mathrm{P}^{*}=0.038048$ (two-tailed) and $\mathrm{P}^{*}=0.019024$ (one-tailed) as seen in Table $3(3)$. There was thus a significant difference in the former at a $1 \%$ or less level of significance, and also a significant difference in the latter at a $5 \%$ or less level of significance. In other words, both degraded carrageenin and aluminum sucrose sulfate were found to exert a similarly excellent effect in terms of the global judgement of effectiveness when compared with lactose, and the former appeared to be slightly superior in effectiveness to the latter. 
TABLE 3. Global judgement made by doctors in charge, and relation between two groups tested by Mann-Whitney Test (U-Test)

(1) Degraded carrageenin group and aluminum sucrose sulfate group

\begin{tabular}{|c|c|c|c|c|c|c|c|}
\hline & $\begin{array}{l}\text { Marlzed- } \\
\text { ly ef- } \\
\text { fective }\end{array}$ & Effective & $\begin{array}{c}\text { Rela- } \\
\text { tively } \\
\text { effective }\end{array}$ & $\begin{array}{c}\text { No } \\
\text { change }\end{array}$ & $\begin{array}{c}\text { Exacer- } \\
\text { bated }\end{array}$ & $\begin{array}{c}\text { Total } \\
(\mathrm{nj})\end{array}$ & $\begin{array}{c}\text { Total of } \\
\text { orders of each } \\
\text { group }\left(R_{j}\right)\end{array}$ \\
\hline $\begin{array}{l}\text { Degraded car- } \\
\text { rageenin group }\end{array}$ & 30 & 20 & 18 & 2 & & 70 & $5,161(73.7)$ \\
\hline $\begin{array}{l}\text { Aluminum } \\
\text { sucrose sulfate } \\
\text { group }\end{array}$ & 29 & 30 & 16 & 5 & 1 & 81 & $6.315(78.0)$ \\
\hline $\begin{array}{l}\text { Total } \\
\text { (cumulative } \\
\text { total) }\end{array}$ & 59 & $\begin{array}{l}50 \\
(109)\end{array}$ & $\begin{array}{l}34 \\
(143)\end{array}$ & $\begin{array}{c}7 \\
(150)\end{array}$ & $\begin{array}{c}1 \\
(151)\end{array}$ & N151 & 11.476 \\
\hline (Average order) & $(30.0)$ & $(84.5)$ & $(126.5)$ & $(147.0)$ & $(151,0)$ & & \\
\hline
\end{tabular}

$\mathrm{Z}_{0}=0.6280 \quad \mathrm{P}=0.5300$ (two-tailed) $\mathrm{P}=0.2650$ (one-tailed)

Result of $U$-test: At $p<0.01$, significant difference in drug was found between degraded carrageenin group and aluminum sucrose sulfate group.

(2) Degraded carrageenin group and lactose group

\begin{tabular}{l|c|c|c|c|c|c|c}
\hline \multicolumn{1}{|c|}{ Group } & $\begin{array}{c}\text { Marked- } \\
\text { ly ef- } \\
\text { fective }\end{array}$ & Effective & $\begin{array}{c}\text { Rela- } \\
\text { tively } \\
\text { effective }\end{array}$ & $\begin{array}{c}\text { No } \\
\text { change }\end{array}$ & $\begin{array}{c}\text { Exacer- } \\
\text { bated }\end{array}$ & $\begin{array}{c}\text { Total } \\
\text { (nj) }\end{array}$ & $\begin{array}{c}\text { Total of } \\
\text { orders of each } \\
\text { group (Rj) }\end{array}$ \\
\hline $\begin{array}{c}\text { Degraded car- } \\
\text { rageenin group }\end{array}$ & 30 & 20 & 18 & 2 & & 70 & $4,567(65.2)$ \\
\hline $\begin{array}{c}\text { Lactose group } \\
\text { Total } \\
\text { (cumulative } \\
\text { total) }\end{array}$ & 24 & 19 & 26 & 16 & 3 & 78 & $6,549(82.8)$ \\
\hline $\begin{array}{c}\text { (Average order) } \\
\text { (27.5) }\end{array}$ & $\begin{array}{c}39 \\
(93)\end{array}$ & $\begin{array}{c}34 \\
(127)\end{array}$ & $\begin{array}{c}18 \\
(148)\end{array}$ & $\begin{array}{c}3 \\
(148)\end{array}$ & 148 & 11,026 \\
\hline
\end{tabular}

$Z_{0}=2.5957^{* *} \quad \mathrm{P}^{* *}=0.009478$ (two-tailed) $\quad \mathrm{P}^{* * *}=0.004739$ (one-tailed)

Result of U-test: At $\mathrm{p}<0.01$, significant difference in drug was found between degraded carrageenín group and lactose group.

(3) Aluminum sucrose sulfate group and lactose group

\begin{tabular}{|c|c|c|c|c|c|c|c|}
\hline Group & $\begin{array}{l}\text { Marked- } \\
\text { ly ef- } \\
\text { fective }\end{array}$ & Effective & $\begin{array}{c}\text { Rela- } \\
\text { tively } \\
\text { effective }\end{array}$ & $\begin{array}{c}\text { No } \\
\text { change }\end{array}$ & $\begin{array}{c}\text { Exacer- } \\
\text { bated }\end{array}$ & $\begin{array}{c}\text { Total } \\
\text { (nj) }\end{array}$ & $\begin{array}{l}\text { Total of } \\
\text { orders of each } \\
\text { group }(\mathrm{Rj})\end{array}$ \\
\hline $\begin{array}{l}\text { Aluminum } \\
\text { sucrose sulfate } \\
\text { group }\end{array}$ & 29 & 30 & 16 & 5 & 1 & 81 & $5,901.5$ \\
\hline Lactose group & 24 & 19 & 16 & 16 & 3 & 78 & $6,818.5$ \\
\hline $\begin{array}{l}\text { Total } \\
\text { (cumulative } \\
\text { total) }\end{array}$ & 53 & $\begin{array}{c}49 \\
(102)\end{array}$ & $\begin{array}{c}32 \\
(134)\end{array}$ & $\begin{array}{c}21 \\
(155)\end{array}$ & $\begin{array}{c}4 \\
(159)\end{array}$ & & 12,720 \\
\hline (Average order) & $(27.0)$ & $(78.0)$ & $(118.5)$ & $(145.0)$ & $(157.5)$ & & \\
\hline
\end{tabular}

$Z_{0}=2.0744 * \quad \mathrm{P}^{*}=0.038048$ (tow-tailed) $\quad \mathrm{P}^{*}=0.019024$ (one-tailed)

Result of U-test: At $p<0.05$, significant difference in drug was found between aluminum sucrose sulfate group and lactose group. 


\section{2) Analysis of each factor evaluation in three groups}

The above-mentioned results were attained by aggregation of the outcomes of various factors such as the findings on x-ray examination and gastroscopy, subjective symptoms and results of fecal occult blood reaction. In order, however, to examine in detail how each of these factors contributed to the global judgement of effectiveness, and also whether or not a difference would occur between degraded carrageenin and aluminum sucrose sulfate in relation to these factors, grouped discrimination analysis (The Theory of Quantification, II) in multivariate analysis was applied. A total of 22 items were taken up as the factorial items, and they were roughly classified to the group of morbidity consisting of 3 factors, viz., inpatient or outpatient, primary or recurrent ulcer, and locality of ulcer (I group of factors), the group of subjective symptoms including 16 symptoms (II group of factors), the group of objective findings consisting of $x$-ray and gastroscopic findings and results of fecal occult blood reaction (III group of factors), and the global judgement of effectiveness (IV group of factors). These factors were further classified to classes, items, and categories, with a total of 117 categories as shown in Table 4 in detail, by the outcome, or by the difference in morbidity. Especially for the three groups of factors, viz., subjective symptoms, objective findings, and global judgement of effectiveness was employed the order grading in five steps, viz., (1) disappearance, (2) improvement, (3) no change (progress without manifestation,) (4) ineffective (without outcome of condition), and (5) exacerbation. Of these grades, the "disappearance" and "improvement" were further classified to (1) 3-step improvement $(\mathrm{HH} \rightarrow-)$, (2) 2-step improvement $(\mathrm{H} \rightarrow+, \# \rightarrow-$ ), and (3) l-step improvement $(\mathrm{H} \rightarrow \mathrm{H}, \mathrm{H} \rightarrow+,+\rightarrow-$ ), taking the time factor in unit of two weeks into consideration. Of the $x$-ray and gastroscopic findings, two factors, viz., changes in ulcer lesion and time trend, were combined to make 15 categories.

In the statistic treatment of the results of the evaluation, if a score of $X j k$ was given to category No. $k$, factorial-item No. $j$, the sample score $Y i$ for a randomly sampled case would be expressed by the following equation:

$$
Y i=\sum_{j}^{R} \sum_{k}^{K j} X i k \cdot \delta i(j k)
$$

where: $\quad \delta i(j k)=1$, when sample No. $i$ corresponds to category No. $k$, item No. $j$. $\delta i(j k)=0$, when sample No. $i$ does not correspond to category No. $k$, item No. $j$.

(R stands for the total number of items, and $k j$ stands for the number of categories in item No. $j$.)

In this case, a randomly sampled category score $X j k$ is numerically expressed so as to minimize the within-class variance in the same group, and to maximize the between-class variance between different groups. This is equal to maximizing the ratio of the within-class variance to the sum of the within class variance and the between-class variance, viz., the total variance, or to maximizing the correlation 
TABLE 4. Discrimination-

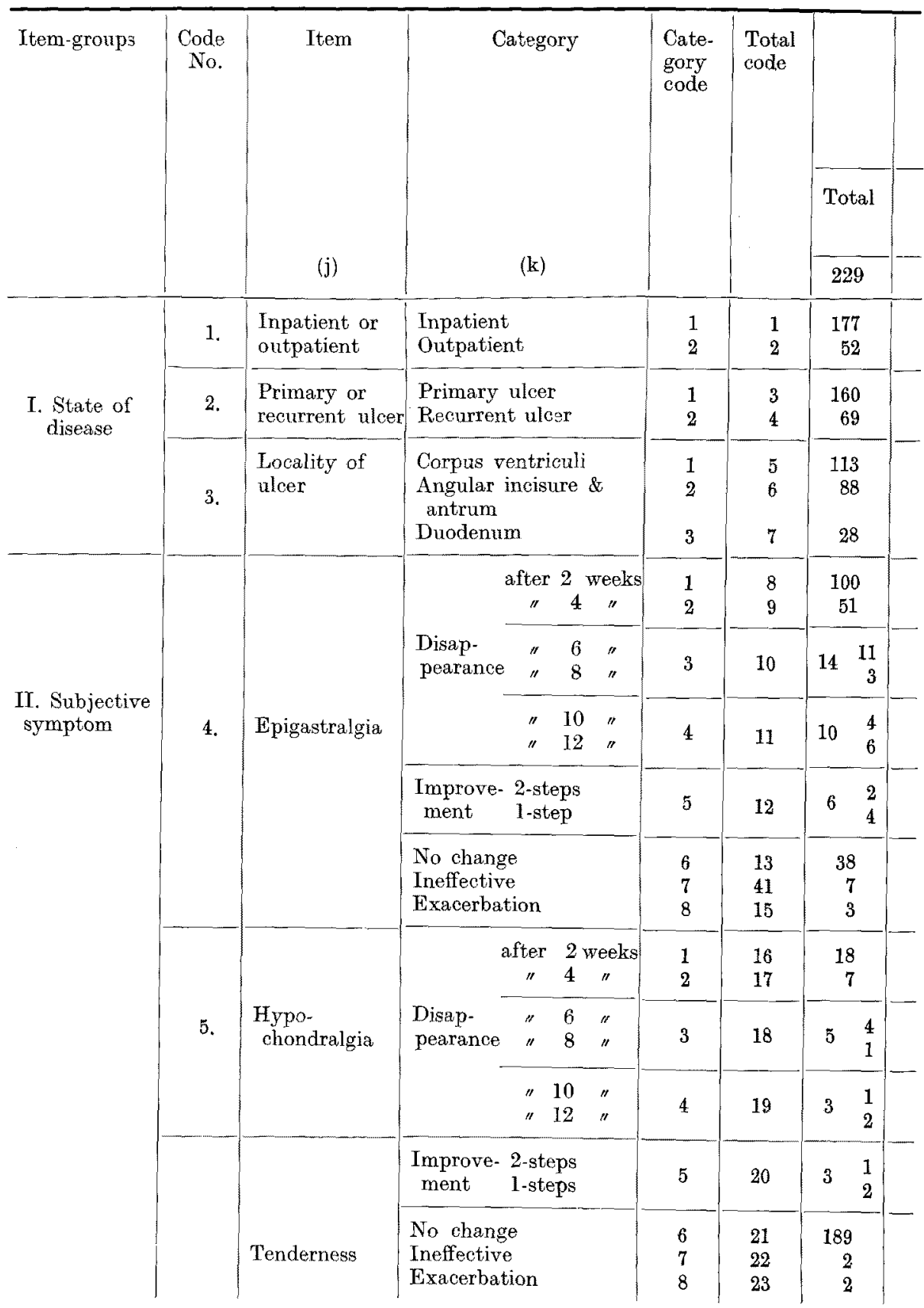


analysis table

\begin{tabular}{|c|c|c|c|c|c|c|}
\hline \multicolumn{3}{|c|}{$\begin{array}{l}\text { Number of patients } \\
\text { actually treated }\end{array}$} & \multicolumn{2}{|c|}{$\begin{array}{l}\text { Radical } 1 . \\
\text { Difference in therapea- } \\
\text { tie effect of the drug } \\
\text { against placebo. }\end{array}$} & \multicolumn{2}{|c|}{$\begin{array}{l}\text { Radical } 2 \text {. } \\
\text { Difference in the mani- } \\
\text { festation of therapeutic } \\
\text { effect between degraded } \\
\text { carrageenin \& aluminum } \\
\text { sucrose sulfate. }\end{array}$} \\
\hline $\begin{array}{l}\text { Degraded } \\
\text { carrageenin } \\
\text { group }\end{array}$ & $\begin{array}{l}\text { Aluminum } \\
\text { sucrose sul- } \\
\text { fate group }\end{array}$ & $\begin{array}{l}\text { Lactose } \\
\text { group }\end{array}$ & \multirow{2}{*}{$\begin{array}{l}\text { Standar- } \\
\text { dized score } \\
\text { (1) } x \text { jh- } \\
\text { (1) } x j\end{array}$} & \multirow{2}{*}{$\begin{array}{l}\text { Partial co- } \\
\text { relation } \\
\text { coefficient } \\
\text { of item. }\end{array}$} & \multirow{2}{*}{$\begin{array}{l}\text { Standard- } \\
\text { ized score } \\
\text { (2) } \mathrm{xjh}- \\
\text { (2) } \mathrm{Xj}\end{array}$} & \multirow{2}{*}{$\begin{array}{l}\text { Partial } \\
\text { correlation } \\
\text { coefficient } \\
\text { of item }\end{array}$} \\
\hline 70 & 81 & 78 & & & & \\
\hline $\begin{array}{l}57 \\
13\end{array}$ & $\begin{array}{l}61 \\
20\end{array}$ & $\begin{array}{l}59 \\
19\end{array}$ & $\begin{array}{r}-0.04567 \\
0.15545\end{array}$ & $0.139^{*}$ & $\begin{array}{r}-0.00551 \\
0.01877\end{array}$ & 0.022 \\
\hline $\begin{array}{l}52 \\
18\end{array}$ & $\begin{array}{l}55 \\
26\end{array}$ & $\begin{array}{l}53 \\
25\end{array}$ & $\begin{array}{r}0.00623 \\
-0.01444\end{array}$ & 0.000 & $\begin{array}{r}-0.01613 \\
0.03741\end{array}$ & 0.042 \\
\hline $\begin{array}{l}34 \\
29\end{array}$ & $\begin{array}{l}38 \\
29\end{array}$ & $\begin{array}{l}41 \\
30\end{array}$ & $\begin{array}{r}-0.01552 \\
0.01741\end{array}$ & \multirow[t]{2}{*}{0.022} & $\begin{array}{l}0.00929 \\
0.00534\end{array}$ & \multirow[t]{2}{*}{0.019} \\
\hline 73 & 14 & 7 & 0.00792 & & 0.02068 & \\
\hline $\begin{array}{l}32 \\
20\end{array}$ & $\begin{array}{l}43 \\
15\end{array}$ & $\begin{array}{l}25 \\
16\end{array}$ & $\begin{array}{r}-0.00895 \\
0.18090\end{array}$ & \multirow{5}{*}{$0.306^{* *}$} & $\begin{array}{r}011665 \\
-0.19885\end{array}$ & \multirow{5}{*}{0.092} \\
\hline $\begin{array}{l}1 \\
1\end{array}$ & $\begin{array}{l}5 \\
1\end{array}$ & $\begin{array}{l}5 \\
1\end{array}$ & -0.11151 & & 0.10192 & \\
\hline $\begin{array}{l}1 \\
1\end{array}$ & 2 & $\begin{array}{l}3 \\
3\end{array}$ & 0.03269 & & -0.01389 & \\
\hline $\begin{array}{l}1 \\
2\end{array}$ & $\begin{array}{l}1 \\
1\end{array}$ & 1 & 0.83844 & & -1.11965 & \\
\hline $\begin{array}{l}8 \\
3\end{array}$ & 13 & $\begin{array}{r}17 \\
4 \\
3\end{array}$ & $\begin{array}{l}-0.19032 \\
-0.51579 \\
-0.42809 \\
\end{array}$ & & $\begin{array}{r}0.13241 \\
-0.13852 \\
-0.05186 \\
\end{array}$ & \\
\hline \multirow[t]{2}{*}{$\begin{array}{l}3 \\
2\end{array}$} & $\begin{array}{r}10 \\
2\end{array}$ & $\begin{array}{l}5 \\
3\end{array}$ & $\begin{array}{r}-0.24237 \\
0.36073\end{array}$ & & $\begin{array}{r}0.10353 \\
-0.13657\end{array}$ & \multirow[b]{3}{*}{0.081} \\
\hline & 1 & $\begin{array}{l}3 \\
1\end{array}$ & -0.59709 & & -0.29540 & \\
\hline \multirow[t]{2}{*}{2} & & 1 & -0.29800 & $0.360^{* *}$ & -0.24363 & \\
\hline & 1 & 2 & -3.45307 & & -1.34289 & \\
\hline 63 & 67 & $\begin{array}{r}59 \\
2 \\
2\end{array}$ & $\begin{array}{l}0.02304 \\
1.67021 \\
4.19066\end{array}$ & & $\begin{array}{r}0.02662 \\
0.42637 \\
4.75056\end{array}$ & \\
\hline
\end{tabular}


TABLE 4.

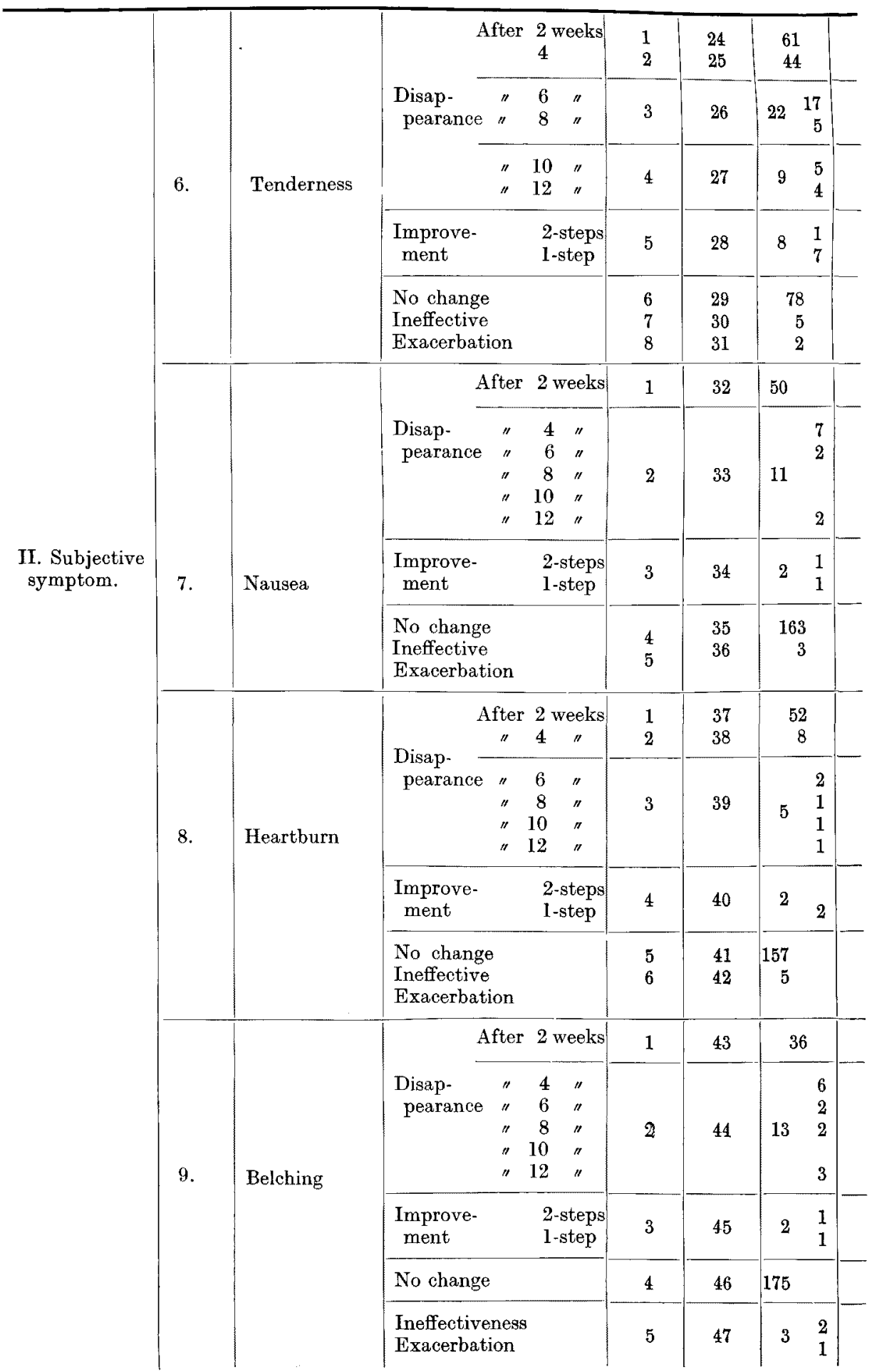


Continued.

\begin{tabular}{|c|c|c|c|c|c|c|}
\hline $\begin{array}{l}19 \\
16\end{array}$ & $\begin{array}{l}27 \\
14\end{array}$ & $\begin{array}{l}15 \\
14\end{array}$ & $\begin{array}{r}0.07221 \\
-0.06807\end{array}$ & \multirow{5}{*}{$0.186^{* *}$} & $\begin{array}{r}-0.00175 \\
0.05718\end{array}$ & \multirow{5}{*}{0.067} \\
\hline $\begin{array}{l}3 \\
1\end{array}$ & $\begin{array}{l}7 \\
1\end{array}$ & $\begin{array}{l}7 \\
3\end{array}$ & -0.02308 & & -0.00877 & \\
\hline $\begin{array}{l}2 \\
1\end{array}$ & $\begin{array}{l}1 \\
1\end{array}$ & $\begin{array}{l}2 \\
2\end{array}$ & 0.03383 & & 0.04703 & \\
\hline 3 & $\begin{array}{l}1 \\
2\end{array}$ & 2 & -0.41344 & & 0.83878 & \\
\hline $\begin{array}{r}24 \\
1\end{array}$ & $\begin{array}{r}26 \\
1\end{array}$ & $\begin{array}{r}28 \\
4 \\
1\end{array}$ & $\begin{array}{r}-0.01157 \\
0.74591 \\
-0.36290\end{array}$ & & $\begin{array}{l}-0.06419 \\
-0.26796 \\
-1.50159\end{array}$ & \\
\hline 16 & 21 & 13 & 0.00485 & \multirow{5}{*}{$0.143^{*}$} & 0.04834 & \multirow{5}{*}{0.133} \\
\hline \multirow{3}{*}{1} & 2 & $\begin{array}{l}3 \\
2\end{array}$ & -0.13224 & & -0.15881 & \\
\hline & & 1 & & & & \\
\hline & 1 & 1 & 0.58397 & & 2.14172 & \\
\hline $\begin{array}{r}50 \\
1\end{array}$ & 57 & $\begin{array}{r}56 \\
2\end{array}$ & $\begin{array}{r}0.02802 \\
-1.50742\end{array}$ & & $\begin{array}{r}0.04346 \\
-4.01251\end{array}$ & \\
\hline $\begin{array}{r}16 \\
4\end{array}$ & $\begin{array}{r}17 \\
2\end{array}$ & $\begin{array}{r}19 \\
2\end{array}$ & $\begin{array}{r}-0.08865 \\
0.36053\end{array}$ & \multirow{4}{*}{$0.183^{* *}$} & $\begin{array}{r}-0.15761 \\
0.30326\end{array}$ & \multirow{4}{*}{0.050} \\
\hline 1 & $\begin{array}{l}1 \\
1\end{array}$ & 2 & 0.09389 & & -0.23982 & \\
\hline 1 & & 1 & 0.31540 & & 0.67983 & \\
\hline $\begin{array}{r}45 \\
4 \\
1\end{array}$ & $\begin{array}{r}60 \\
2 \\
1\end{array}$ & $\begin{array}{r}52 \\
2\end{array}$ & $\begin{array}{r}-0.01761 \\
0.67782\end{array}$ & & $\begin{array}{r}0.05133 \\
-0.54618\end{array}$ & \\
\hline 8 & 16 & 12 & -0.16087 & \multirow{6}{*}{$0.286^{* *}$} & 0.12430 & \\
\hline $\begin{array}{l}3 \\
1 \\
2\end{array}$ & $\begin{array}{l}1 \\
1\end{array}$ & 2 & 0.44955 & & -0.03024 & \multirow{5}{*}{0.055} \\
\hline 1 & 2 & & & & & \\
\hline & 1 & 1 & 1.94667 & & 1. 29144 & \\
\hline \multirow[t]{2}{*}{55} & 60 & 60 & -0.01387 & & -0.04278 & \\
\hline & & $\begin{array}{l}2 \\
1\end{array}$ & -0.50642 & & 0.27406 & \\
\hline
\end{tabular}


TABLE 4

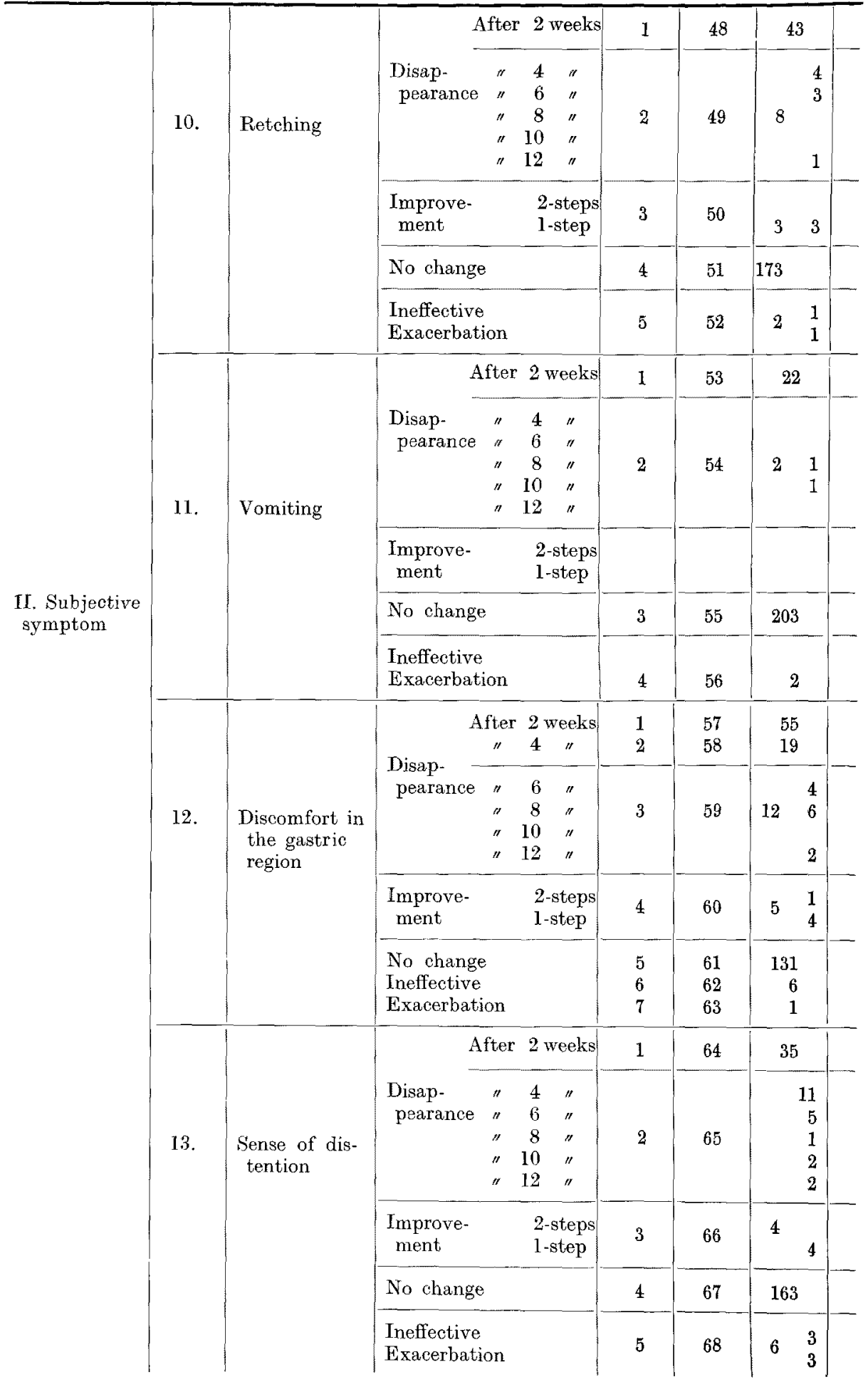


Clinical Evaluation of Pharmacotherapy of Peptic Ulcer

\begin{tabular}{|c|c|c|c|c|c|c|}
\hline 10 & 19 & 14 & 0.02646 & \multirow{6}{*}{$0.218^{* *}$} & 0.00759 & \multirow{6}{*}{0.126} \\
\hline 2 & 1 & $\begin{array}{l}2 \\
2\end{array}$ & -0.32156 & & -0.74823 & \\
\hline 1 & & & & & & \\
\hline 1 & 1 & 1 & 1. 13404 & & 2.44736 & \\
\hline \multirow[t]{2}{*}{56} & 59 & 58 & -002704 & & -0.04338 & \\
\hline & 1 & & 1.35558 & & 2.91116 & \\
\hline 4 & 12 & 6 & -0.18350 & \multirow{4}{*}{$0.357^{* *}$} & 0.37305 & \multirow{4}{*}{0.092} \\
\hline 1 & & & 1. 69598 & & -0.93384 & \\
\hline \multirow[t]{2}{*}{65} & 68 & 70 & 0.03624 & & 0.00246 & \\
\hline & 1 & 6 & -3.35606 & & -3.41966 & \\
\hline $\begin{array}{r}18 \\
4\end{array}$ & $\begin{array}{r}24 \\
6\end{array}$ & $\begin{array}{r}13 \\
9\end{array}$ & $\begin{array}{r}0.06287 \\
-0.10710\end{array}$ & \multirow{5}{*}{$0.204^{* *}$} & $\begin{array}{l}-0.04801 \\
-0.10649\end{array}$ & \multirow{5}{*}{0.023} \\
\hline & $\begin{array}{l}1 \\
3\end{array}$ & $\begin{array}{l}3 \\
3\end{array}$ & -0.31525 & & 0.17685 & \\
\hline 1 & 1 & & & & & \\
\hline 1 & 1 & 3 & -0.27046 & & 0.40231 & \\
\hline $\begin{array}{r}44 \\
2\end{array}$ & $\begin{array}{r}44 \\
2\end{array}$ & $\begin{array}{r}43 \\
3 \\
1\end{array}$ & $\begin{array}{r}0.02067 \\
0.30886 \\
-0.84835\end{array}$ & & $\begin{array}{r}0.00038 \\
0.15627 \\
-0.45685\end{array}$ & \\
\hline 12 & 17 & 6 & 0.12200 & \multirow{5}{*}{$0.240^{* *}$} & 0.11186 & \multirow{5}{*}{0.055} \\
\hline $\begin{array}{l}3 \\
1\end{array}$ & $\begin{array}{l}4 \\
1 \\
1 \\
1\end{array}$ & $\begin{array}{l}4 \\
3 \\
1 \\
1\end{array}$ & -0.26778 & & 0.36766 & \\
\hline & 1 & 3 & -0.82571 & & -0.55023 & \\
\hline 51 & 56 & 56 & 0.01681 & & -0.03770 & \\
\hline $\begin{array}{l}1 \\
1\end{array}$ & & $\begin{array}{l}2 \\
2\end{array}$ & 0.31978 & & -0.54843 & \\
\hline
\end{tabular}


TABLE 4.

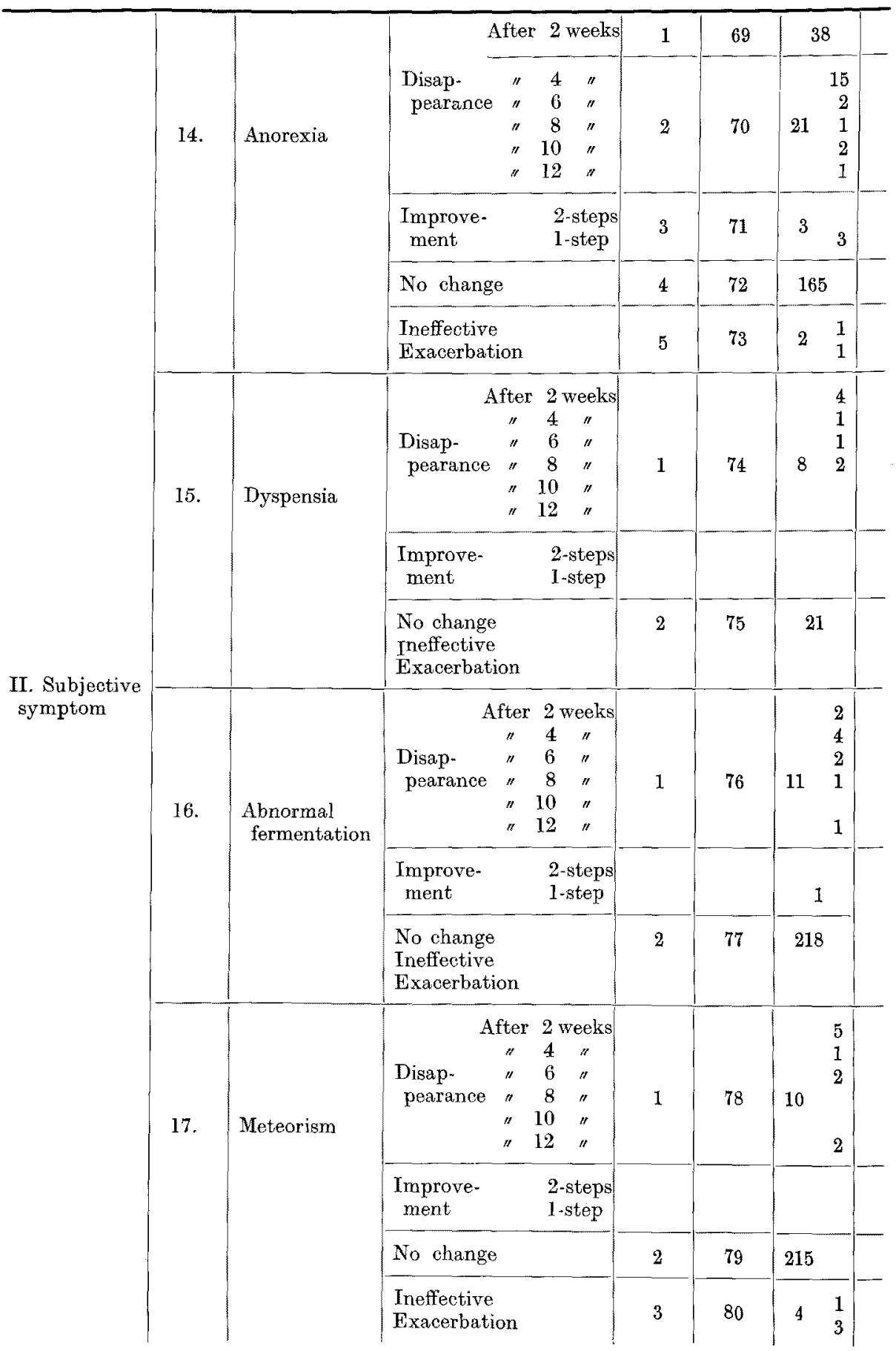


Continued

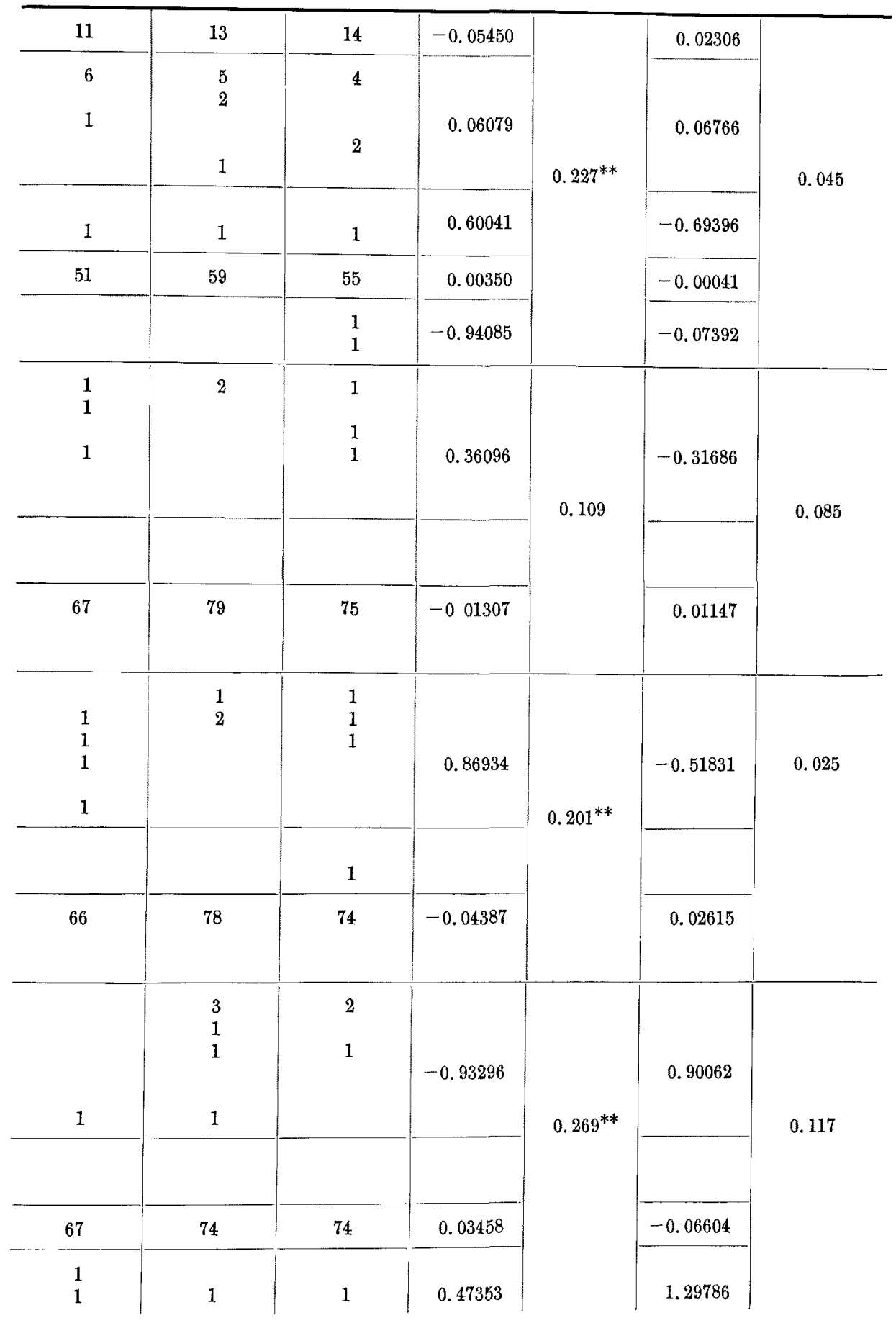


Table 4.

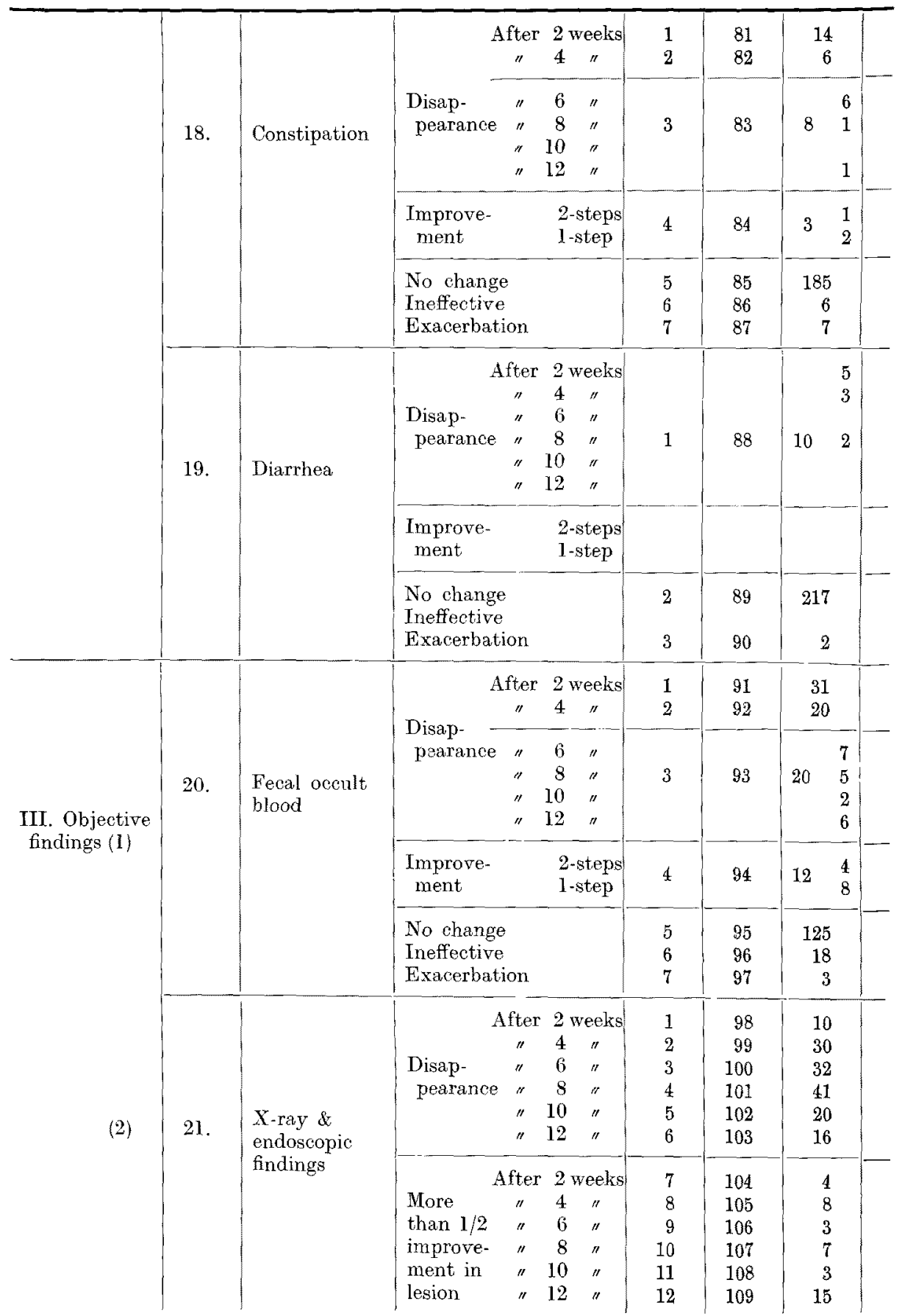


Continued

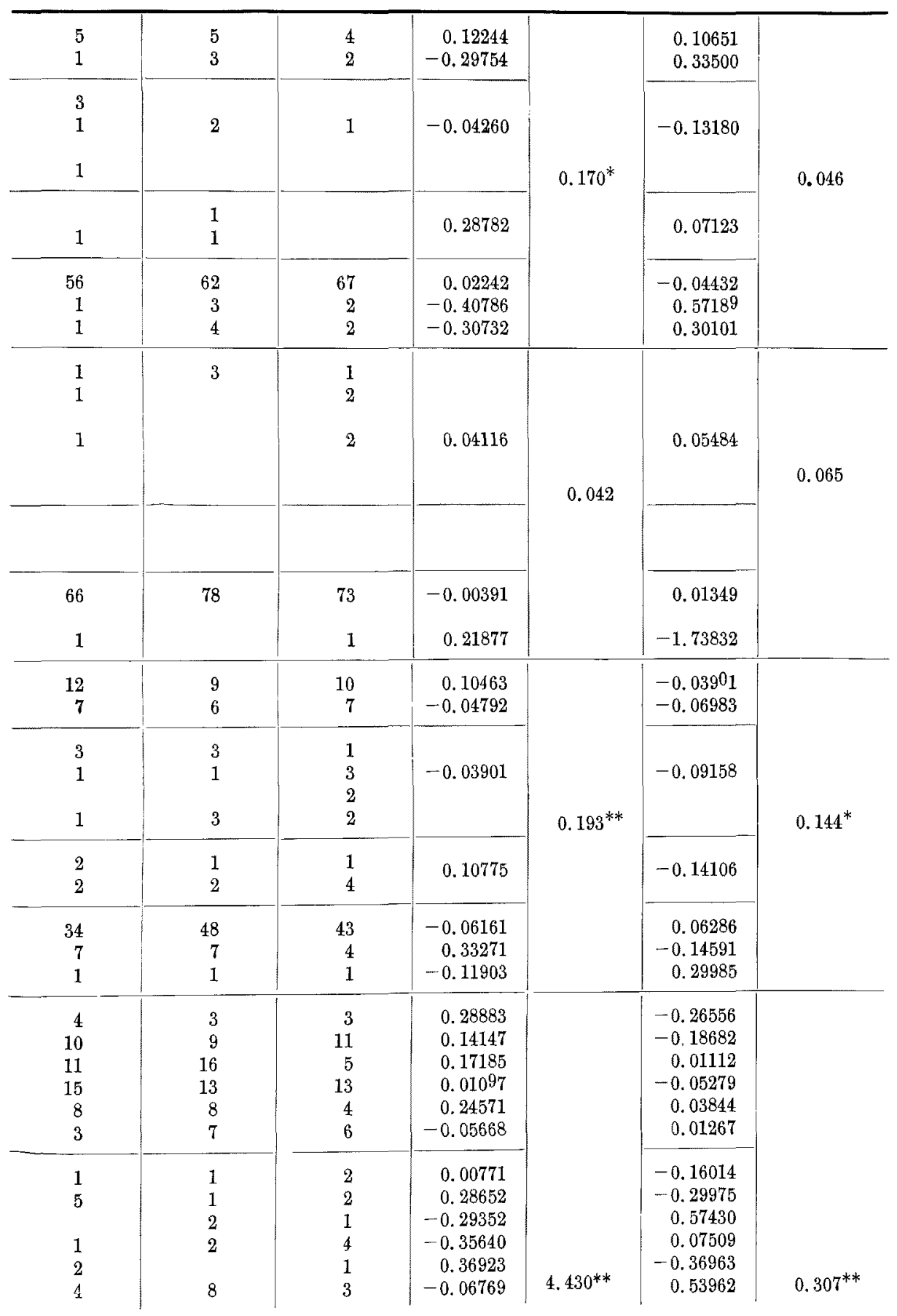


TABle 4.

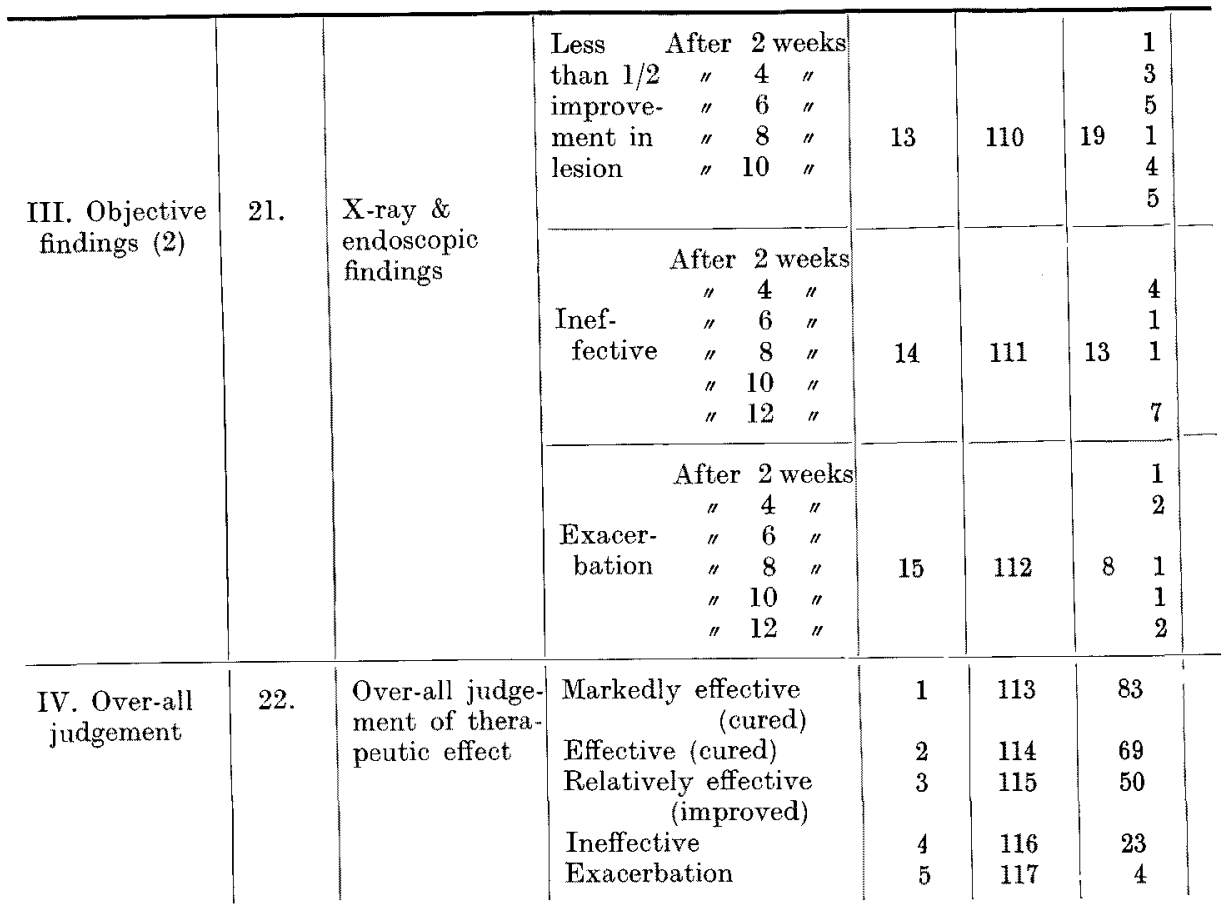

ratio $\eta$ given by the following equation:

$$
\eta=\sqrt{\frac{\sum_{t}^{\tau} n t(\bar{y} t-\bar{y})^{2}}{N \sigma^{2}}}
$$

where

$$
0 \leqq \eta \leqq 1
$$

$$
\sigma^{2}=\frac{1}{N} \sum_{i}^{N}(\bar{y} i-\bar{y})^{2} \quad \text { total variance. }
$$

$T$ stands for the total number of medicated groups, viz., 3 .

$$
\sum_{t}^{\tau} n t=N, \text { total number of cases. }
$$

$\bar{y}$ stands for the mean of all sample scores, which is designated as 0 .

$n t$ stands for the number of cases in each group.

$\bar{y} t$ stands for the mean of scores in group No. $t$.

In practice, the standardized score $(x j k-\bar{x} j)$ can be obtained by deducting the frequency distribution mean $\operatorname{score}\left(\bar{x} j=\sum_{j}^{K j} n j k \cdot X j k / N\right)$ of the factor, and the standardized scores so obtained are best for distinguishing the difference between the medicated groups, and shown in Table 4.

Attention should be directed, however to the fact that the category in the 
Continued

\begin{tabular}{|c|c|c|c|c|c|c|}
\hline $\begin{array}{l}1 \\
2 \\
\end{array}$ & $\begin{array}{l}1 \\
2 \\
1 \\
1 \\
2 \\
1\end{array}$ & $\begin{array}{l}1 \\
2\end{array}$ & 0.01233 & \multirow[t]{3}{*}{$0.430^{* *}$} & 0.14680 & \multirow[t]{3}{*}{$0.327^{* *}$} \\
\hline \multirow[t]{2}{*}{1} & $\begin{array}{l}1 \\
1\end{array}$ & $\begin{array}{l}1 \\
7\end{array}$ & 0.03527 & & -0.01531 & \\
\hline & 1 & $\begin{array}{l}1 \\
2 \\
1 \\
1 \\
2\end{array}$ & -2.10115 & & 0.03897 & \\
\hline 30 & 29 & 24 & 0.02338 & & 0.08229 & \\
\hline $\begin{array}{l}20 \\
18\end{array}$ & $\begin{array}{l}30 \\
16\end{array}$ & $\begin{array}{l}19 \\
16\end{array}$ & $\begin{array}{l}0.02334 \\
0.02587\end{array}$ & $0.327^{* *}$ & $\begin{array}{r}0.12393 \\
-0.15101\end{array}$ & 0.059 \\
\hline 2 & $\begin{array}{l}5 \\
1\end{array}$ & $\begin{array}{r}16 \\
3\end{array}$ & $\begin{array}{l}-0.17869 \\
-0.18370\end{array}$ & & $\begin{array}{l}-0.19979 \\
-0.80879\end{array}$ & \\
\hline
\end{tabular}

item is the order grade which expresses the outcome, and the standardized scores also follow the order, and the size of absolute value of the sample score for each case primarily expresses the quality. And if the correlation ratio $\eta$ was tested to be of significance below a given level of significance, it would mean that a significant difference existed in the set up item between the groups. Further, once the ratio was expressed numerically, it would be possible to make statistic calculation of it, and the partial correlation coefficients of item for showing the rate of contribution of each factor to discrimination were also calculated as shown in Table 4 . If the figures were found significant, it would be that the figures have contributed to the discrimination in the order thereof.

In the discrimination of three medicated groups, two groups are placed so as to maximize the correlation ratio $\eta$ which shows discrimination efficiency, and after it is made clear where another group would be plotted, the combination of two groups is changed, viz., one of the first two groups and the last group are placed, and it is determined where the excluded one group would be plotted. Radical 1 in Table 4 was obtained by such a means that the degraded carrageeningiven group and lactose-given group were placed, and it was calculated where the group given aluminum sucrose sulfate would be placed when the discrimination was maximized. It may, therefore, be considered to represent the difference in therapeutic effect, whereas Radical 2 was obtained by such a means that the degraded carrageenin-given group and the group given aluminum sucrose sulfate 


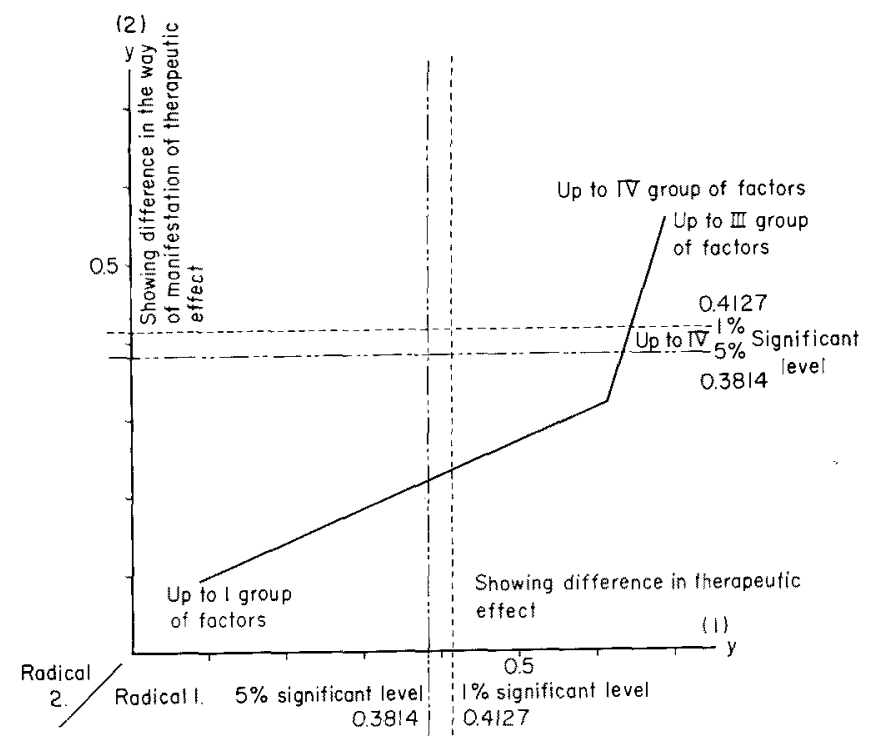

Fig. 1. Sequential partial correlation ratio of item-groups.

were first placed, and it may be considered to represent the different manifestations of therapeutic effects.

As shown in Fig. 1, the results of discrimination analysis were such that the sequential correlation ratio of item-groups was 0.1238 and 0.0786 for both Radicals 1 and 2 up to I group of factors which represent morbidity, showing no significant difference at a $5 \%$ level of significance. It indicates that there was no difference in therapeutic effect among the medicated groups, with such a factor as inpatient or outpatient, primary or recurrent ulcer, or locality of ulcer. It demonstrated also that the random arrangement was effectively made under the double blind technique employed.

When II group of factors consisting of 16 subjective symptoms was added, the sequential correlation ratio of the item-groups became 0.6036 for Radical 1, with significance at a $1 \%$ or less level of significance, but it definitely rose for Radical 2 , though up to 0.2712 as a maximum, gaining no significance. In other words, both degraded carrageenin and aluminum sucrose sulfate were found to show a distinct difference in therapeutic effect from lactose, in terms of the improvement of subjective symptoms.

When III group of items consisting of objective findings was further added, the sequential correlation ratio substantially increased for both Radicals 1 and 2, to 0.6572 and 0.5737 , respectively, with significance at a $1 \%$ or less level of significance, and thus showed the existance of a significant difference among the three medicated groups. In other words, the objective findings largely contributed to discrimination among the three medicated groups either in terms of the difference in effectiveness or the difference in the way of manifestation of effect, viz., it was clear that the active agents were effective also in terms of the objective findings. 
In the last place, when the statistical treatment was similarly made by adding all factors up to IV group of factors for the global judgement of effectiveness, viz., all 22 factors, the ratio somewhat fell to 0.6541 for Radical 1 but with no significant fall, and along with the ratio of 0.5831 for Radical 2, proved a significant difference existing among the three medicated groups at a $1 \%$ or less level of significance. In other words, the global judgement of effectiveness may be considered to have been aggregately made by the physicians in charge, including other information than the 22 factors, and with reference to their own experience. But the aforesaid results of the evaluation well reflect the results of discrimination of I to III groups of items and may be considered to be so-to-speak "making assurance doubly sure". Different from Radical 1, Radical 2 which represents the difference in the way of manifestation of effect did not gain significance in the sequential correlation ratio of up to II group of factors, viz., subjective symptoms; in other words, no difference was found to exist between degraded carrageenin and aluminum sucrose sulfate in the improvement of subjective symptoms. The sequential correlation ratio of up to IIJ group of items viz., objective findings, however, gained significance very much, and the difference in the way of manifestation of effect between the two active agents could be considered attributable to the objective findings.

In order to get hold of the status of discrimination of Radicals 1 and 2 simultaneously and aggregately among the three medicated groups, the sample score in each case was plotted on a two-dimensional discrimination graph (Fig. 2). The three ellipses are drawn with the mean of sample scores of the respective medicated groups at the centre, showing the sphere of \pm 2 standard deviation times each

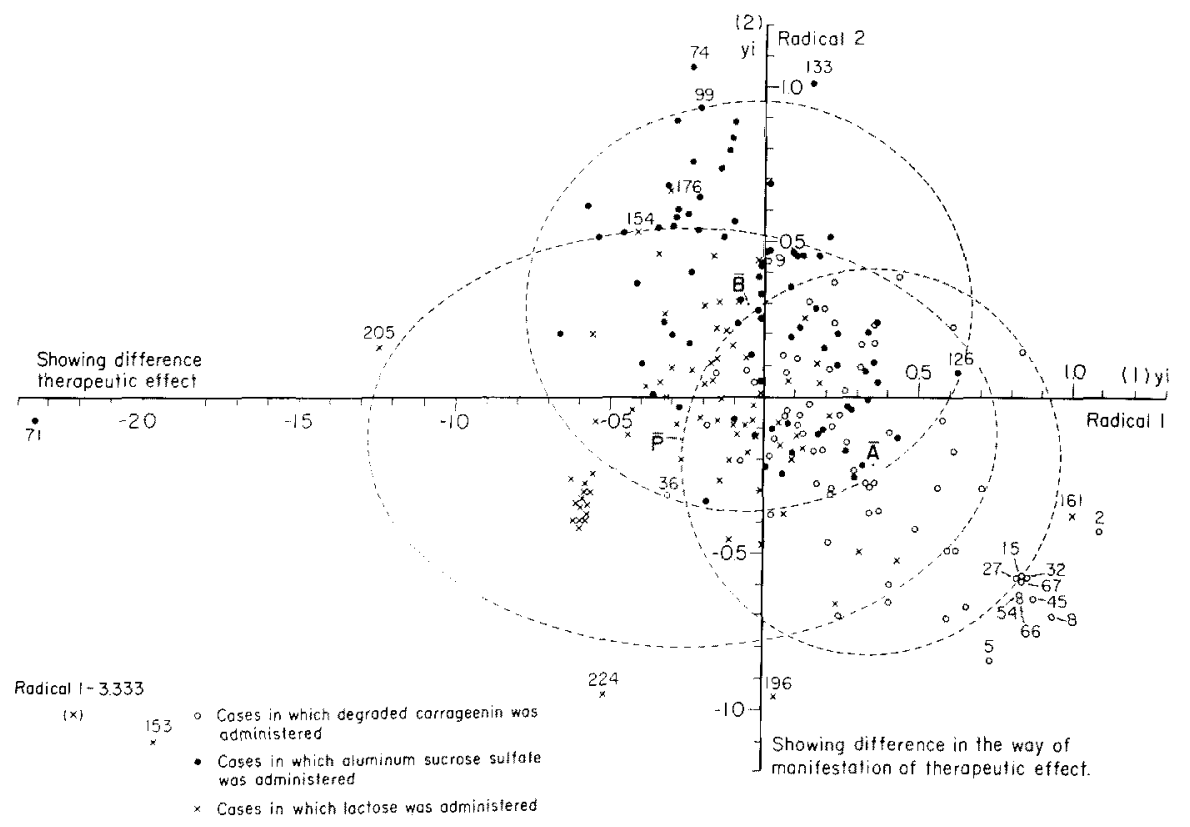

Fig. 2. Two-dimensional discrimination graph. 
radical, and about $72 \%$ of the cases in the respective medicated groups are included in this sphere.

As shown in the foregoing, both degraded carrageenin and aluminum sucrose sulfate were proved to be therapeutically effective, and the two agents were also shown to differ from each other in the way of manifestation of effect in terms of objective symptoms. In order to find what a factor or factors were responsible for such outcomes, the partial correlation coefficient of item was examined by means of discrimination analysis of all 22 factors. And as shown in Table 4, Radical 1 which represents the difference in therapeutic effect was found to be of significance at a $5 \%$ or less level of significance in as many as 18 out of the 22 factors, and it may be considered that a therapeutic effect was manifest on these 18 factors. It

TaBLE 5. Discrimination of haspitalized patient and out patient, and judgement of therapeutic effect

(1) All cases

\begin{tabular}{|c|c|c|c|c|c|c|}
\hline & (Total) & $\begin{array}{l}\text { Markedly } \\
\text { effective }\end{array}$ & Effective & $\begin{array}{c}\text { Relatively } \\
\text { effective }\end{array}$ & $\begin{array}{c}\text { No } \\
\text { change }\end{array}$ & Exacerbated \\
\hline Hospitalized & (nI) 177 & 71 & 56 & 33 & 14 & 3 \\
\hline Out-patient & (n2) 52 & 12 & 13 & 17 & 9 & 1 \\
\hline
\end{tabular}

(2) Degraded carrageenin group

\begin{tabular}{c|c|c|c|c|c|c}
\hline & (Total) & $\begin{array}{c}\text { Markedly } \\
\text { effeotive }\end{array}$ & Effective & $\begin{array}{c}\text { Relatively } \\
\text { effective }\end{array}$ & $\begin{array}{c}\text { No } \\
\text { change }\end{array}$ & Exacerbated \\
\hline Hospitalized & 57 & 26 & 17 & 13 & 1 & \\
\hline Out-Patient & 13 & 4 & 3 & 5 & 1 &
\end{tabular}

U.Test $Z_{0}{ }^{\prime}=1.4319$ (Two-tailed) $\mathrm{p}=0.1522$

(3) Aluminum sucrose sulfate group

\begin{tabular}{c|c|c|c|c|c|c}
\hline & (Total) & $\begin{array}{c}\text { Markedly } \\
\text { effective }\end{array}$ & Effective & $\begin{array}{c}\text { Relatively } \\
\text { effective }\end{array}$ & $\begin{array}{c}\text { No } \\
\text { change }\end{array}$ & Exacerbated \\
\hline Hospitalized & 61 & 26 & 23 & 9 & 2 & 1 \\
\hline Out-patient & 20 & 3 & 7 & 7 & 3 &
\end{tabular}

U-Test $\mathrm{Z}_{0}{ }^{\prime}=2.8593^{* * *}$ (Two-tailed) $\mathrm{p}=0.0042$

(4) Lactose group

\begin{tabular}{c|c|c|c|c|c|c}
\hline & (Total) & $\begin{array}{c}\text { Markedly } \\
\text { effective }\end{array}$ & Effective & $\begin{array}{c}\text { Relatively } \\
\text { effective }\end{array}$ & $\begin{array}{c}\text { No } \\
\text { change }\end{array}$ & Exacerbated \\
\hline Hospitalized & 59 & 19 & 16 & 11 & 11 & 2 \\
\hline Out-patient & 19 & 5 & 3 & 5 & 5 & 1
\end{tabular}

U-Test $\mathrm{Z}_{0}{ }^{\prime}=1.0258$ (Two-tailed) $\mathrm{p}=0.3050$ 
is, however, noteworthy that the item of inpatient or outpatient, which belongs to I group of items and showed no significance in terms of the sequential correlation ratio as shown in Fig. 1, gained significance here. Althongh there was no difference in the distribution of inpatients and outpatients as a single factor among the three medicated groups, this factor was closely correlated with subjective symptoms and objective findings which show a difference in therapeutic effect and further correlated with various factorial-items for the global judgement of effectiveness; in other words, it is nothing but an indication that there was a difference in effectiveness between inpatient and outpatient.

In order to examine this effect, the global judgement of effectiveness was made on categories of inpatient and outpatient in all cases, degraded carrageenin

TABLE 6. Simple correlation coefficient among items

\begin{tabular}{|c|c|c|c|c|c|}
\hline Item groups & Item & $\begin{array}{l}\text { 21. X-ray \& } \\
\text { gastroscopic } \\
\text { findings }\end{array}$ & $\begin{array}{l}\text { 22. Over-all } \\
\text { Judgement }\end{array}$ & $\mid \begin{array}{c}\text { Score of } \\
\text { discrimination } \\
\text { (1) }\end{array}$ & $\begin{array}{c}\text { Score of } \\
\text { discrimination } \\
(2)\end{array}$ \\
\hline $\begin{array}{l}\text { 1. State of } \\
\text { disease }\end{array}$ & $\begin{array}{l}\text { 1. Inpatient or } \\
\text { outpatient } \\
\text { 2. Primary or } \\
\text { recurrent ulcer } \\
\text { 3. Locality of ulcer }\end{array}$ & $\begin{array}{c}-0.12640 \\
0.17582^{* * *} \\
0.04401\end{array}$ & $\begin{array}{r}-0.12203 \\
0.11460 \\
0.03835\end{array}$ & $\begin{array}{r}-0.06014 \\
0.05962 \\
0.02866\end{array}$ & $\begin{array}{l}0.04304 \\
0.03977 \\
0.07602\end{array}$ \\
\hline $\begin{array}{l}\text { II. Sub- } \\
\text { jective } \\
\text { symptom }\end{array}$ & $\begin{array}{l}\text { 4. Epigastralgia } \\
\text { 5. Hypochondralgia } \\
\text { 6. Tenderness } \\
\text { 7. Nausea } \\
\text { 8. Heartbum } \\
\text { 9. Belching } \\
\text { 10. Retching } \\
\text { 11. Vomiting } \\
\text { 12. Discomfort in the } \\
\text { gastric region } \\
\text { 13. Sense of distention } \\
\text { 14. Anorexia } \\
\text { 15. Dyspepsia } \\
\text { 16. Abnormal fermen- } \\
\text { tation } \\
\text { 17. Meteorism } \\
\text { 18. Constipation } \\
\text { 19. Diarrhea }\end{array}$ & $\begin{array}{c}-0.01934 \\
-0.31918^{* *} \\
-0.39703^{* *} \\
0.37220^{* *} \\
-0.26774^{* *} \\
-0.05192 \\
-0.42196^{* *} \\
0.07774 \\
-0.06413 \\
-0.04676 \\
0.16159^{*} \\
-0.09342 \\
-0.16685^{*} \\
0.01506 \\
-0.02935 \\
-0.02237\end{array}$ & $\begin{array}{c}0.03965 \\
-0.11270 \\
-0.21707^{* *} \\
0.14730 \\
-0.09530 \\
-0.11364 \\
-0.32327^{* *} \\
0.17129^{* *} \\
0.07933 \\
-0.05678 \\
0.14046^{*} \\
-0.00459 \\
0.01712 \\
-0.02465 \\
0.07306 \\
0.00970\end{array}$ & $\begin{array}{r}0.15217^{*} \\
-0.01080 \\
-0.11389 \\
0.01556 \\
0.08956 \\
0.05714 \\
-0.03965 \\
0.06780 \\
0.15367^{*} \\
\\
0.12903 \\
0.08709 \\
0.01579 \\
0.01667 \\
\\
0.07515 \\
0.06238 \\
0.01030\end{array}$ & $\begin{array}{r}0.09627 \\
-0.03865 \\
0.04761 \\
0.09204 \\
0.03616 \\
0.05364 \\
0.05198 \\
0.02540 \\
-0.02323 \\
\\
0.08706 \\
0.01199 \\
0.04225 \\
0.03928 \\
\\
0.06284 \\
0.11650 \\
0.06833\end{array}$ \\
\hline $\begin{array}{l}\text { III. } \\
\text { Objective } \\
\text { findings }\end{array}$ & $\begin{array}{l}\text { 20. Fecal occult blood } \\
\text { 21. X-ray \& } \\
\text { endoscopic findings }\end{array}$ & $\begin{array}{l}-0.04288 \\
\quad 1 .\end{array}$ & $\begin{array}{l}-0.03356 \\
0.44478^{* *}\end{array}$ & $\begin{array}{l}0.09050 \\
0.23141^{* *}\end{array}$ & $\begin{array}{l}0.05498 \\
0.17886^{* *}\end{array}$ \\
\hline $\begin{array}{l}\text { IV. Over-all } \\
\text { judgement }\end{array}$ & $\begin{array}{l}\text { 22. Over-all judgement } \\
\text { of therapeutic } \\
\text { effect }\end{array}$ & $0.44478^{* * *}$ & 1. & 0.24946 & 0.07531 \\
\hline $\begin{array}{l}\text { Score of } \\
\text { discriminati }\end{array}$ & & $0.23141^{* *}$ & $0.24946^{* *}$ & 1. & 1. \\
\hline
\end{tabular}

Radical 1. Showing difference in therapeutic effect on the drug against placebo

Radical 2. Showing difference in the manifestation of therapentic effect between degraded carrageenin and aluminum sucrose sulfate. 
cases, aluminum sucrose sulfate cases, and lactose cases respectively (Table 5). A significant difference was found to exist between inpatients and outpatients among all cases at a $0.05 \%$ or less level of significance when tested by the U-test. Accordingly it could be considered that effects of the agents were more liable to manifest in inpatients than in outpatients. The investigation of effect in inpatients and outpatients, however, showed that there was no significant difference between them in the degraded carrageenin group, and it could be considered that degraded carrageenin was effective regardless of inpatient or outpatient. But a large, significant difference was present between inpatients and outpatients in the group given aluminum sucrose sulfate, which was interpreted that the therapeutic effect was more liable to manifest in inpatients than in outpatients.

In order to examine the status of contribution of various factorial-items on which basis the global judgement of effectiveness was made by each physician in charge, a test for significance was made on the simple correlation coefficient among items. As shown in Table 6, among the factors of Radical 1 which shows difference in therapeutic effect, those which were proved to have significant correlation with the results of the global judgement of effectiveness at a $5 \%$ or less level of significance were such as $\mathrm{x}$-ray and gastroscopic findings, ${ }^{* *}$ retching, ${ }^{* *}$ tenderness, ${ }^{* *}$ vomiting, ${ }^{* *}$ nausea** and anorexia** in the sequence of values. Therefore each physician may be said to have made the evaluation of effectiveness based on the outcomes of these 7 findings and symptoms.

As to the between-factor simple correlation coefficient between the $x$-ray and endoscopic findings which are most closely related with the global judgement of effectiveness and various factorial-items, it was noteworthy that the $x$-ray and endoscopic findings were correlated with the manifestation of I group of factors, viz., the item of primary or recurrent ulcer. Judging from the standardized score of Radical 1 of both items shown in Table 4, it may be interpreted to indicate that primary ulcer is liable to disappear in terms of the $x$-ray and endoscopic findings.

Each factor of Radical 2 which represents the difference in manifestation of therapeutic effect was found not to show a significant difference from the results of the global judgement of effectiveness, but only the discrimination score with which each case is plotted and the $x$-ray and endoscopic findings were closely correlated.

No noticeable side-effects were reported throughout the entire study.

\section{Discussion}

In relation to the pharmacotherapy for peptic ulcer it has been stressed that the digestive power of gastric juice is due not to acid but rather to pepsin, and most antiulcer drugs may contribute to healing of peptic ulcer through lessening the activity of pepsin in various ways. Babkin and Komarov in 1932 found that such substances as chondroitin sulfate contained in gastric mucus directly inhibit the proteolytic action of pepsin irrespective of acid, and they predicted the possi- 
bility of the substances being used for the treatment of peptic ulcer in the future, and attention has since then been directed to the therapy of peptic ulcer with antipepsin agents. A variety of anionic carbohydrates has been developed as antipepsin agents and studies have been made in detail of the correlations of their chemical structures to the antipeptic action or of their mechanisms of action (Levey and Sheinfeld 1954; Anderson 1961; Cook et al. 1963; Martin et al. 1968; Ishimori 1971) and some of them are already in clinical use like aluminum sucrose sulfate in Japan.

Degraded carrageenin is also one of antipepsin agents, which has shown in animal experiments to have an excellent protective action against the development of ulcer caused by histamine, ligation of the pylorus, or cortisone (Anderson and Watt 1959; Houck et al. 1960). Unlike antacids antipepsin agents are expected to display their clinical effectiveness for peptic ulcer even without combined use of other antiulcer drugs according to its mode of action (Ishimori 1971). This is the reason for that these antipepsin agents were adopted in the present clinical study by the double blind technique.

As already pointed out, no standardized method has been available for the clinical evaluation of the therapeutic effect of antiulcer agents because of various difficulties involved, and the results reported in many studies have been regarded as low in reliability (Piper 1967). It is most probable for the reason that the cause of peptic ulcer or the mechanism of ulcer becoming ehronic has not been elucidated in full. This means that in general the tendency toward spontaneous healing or the close cause-and-effect relation between various circumstantial, psychological or physical factors and the course of disease has not been amply understood in peptic ulcer, and it also implies that all patients of peptic ulcer cannot be handled as the subjects of uniformity or at the same level in respect of their courses or prognoses. It is, therefore, clear that, in order to accurately get hold of the clinical effect of a pharmacotherapy for peptic ulcer by a scientific means, it is the method of solution that can be first thought of, to overcome the fact that the patients of peptic ulcer are not uniform in course and prognosis. It is the method that meets the above-mentioned requisitions to compare the therapeutic effects of the test agent with that of lactose or those of an agent the therapeutic effect of which has already been demonstrated clinically, and to statistically examine the results. The evaluation of the therapeutic effect of the test agent, degraded carrageenin was therefore made by the double blind technique at as many medical institutes as 13 all over Japan, with lactose as the inactive placebo, and with aluminum sucrose sulfate which belongs to the group of antipepsin agent as the control agent.

The criteria of evaluation are one of the most important problems in the clinical evaluation of the therapeutic effects of an agent, and it is also important to standardize the criteria beforehand in such experiments that will be made at many different institutes.

The experiments shown in the present paper are featured in that the criteria 
of evaluation were standardized in such a manner that the $x$-ray examination and gastroscopy were repeated at two-week intervals to follow up the change of ulcer lesion, and in cases of gastric ulcer the endoscopically confirmed disappearance of white coating was regarded to represent the state of healing. The superiority of endoscopy to x-ray examination is doubtless in the judgement of healing of ulcer. The evaluation of therapeutic effect in the experiments shown in the present paper may, therefore, be pointed out to be markedly higher in objectivity and reliability when compared with the studies in which only the $\mathrm{x}$-ray findings and subjective symptoms were followed up (Lawrence 1952; Doll et al. 1956; Sun 1964; Zimmon et al. 1964; Doll et al. 1965; Horwich and Galloway 1965), since most of our patients were diagnosed as gastric ulcer, that is only 28 out of 229 cases subjected to analysis were reported as duodenal ulcer.

In the statistical analysis of the results, aggregate non-parametric test was made for the global judgement by the physicians in charge, and both degraded carrageenin and aluminum sucrose sulfate were proved to have a significant therapeutic effect against lactose. At the same time, grouped discrimination analysis in the multivariate analysis was applied to the 22 factors set up as criteria of assessment of therapeutic effect so as to find how each factor contributed to the results of the global judgement of effectiveness. And consequently, the x-ray and gastroscopic findings were proved to be most closely correlated with, and to exert the largest influence on the results of the global judgement. This may be interpreted to imply that the results reported in the present paper are very objective in nature, and very reliable, accordingly.

There were five symptoms, viz., retching, tenderness, vomiting, nausea, and anorexia, which may be considered to have largely influenced the global judgement of effectiveness, in addition to the $\mathrm{x}$-ray and gastroscopic findings. These five symptoms are generally accepted to be closely correlated with the $\mathrm{x}$-ray and endoscopic findings, and in view of the fact that the course of ulcer lesion and the subjective symptoms are not totally independent but closely correlated with each other, it may be considered a very reasonable sequence.

What was most noticeable in relation to it was the fact that the $x$-ray and gastroscopic findings which are mostly closely correlated with the global judgement of effectiveness were shown to be correlated with the type of manifestation, one of the factors of morbidity, viz., category of primary or recurrent ulcer. And as it has already been pointed out from the standardized score of Radical 1 of both tactors shown in Table 4, "primary peptic ulcer" may be interpreted to be an ulcer easily curable. It is usually difficult to distinguish primary from recurrent ulcer endoscopically or roentgenologically. Although it is recognized that the rate of recurrence is remarkably high in peptic ulcer and many ulcer patients may be asymptomatic providing that the past history as to recurrence obtained from individual patient may be not so reliable, it is to be noted that the results shown in the present study support the up to date long held general clinical impression. On the other hand, whether the case was an inpatient or an outpatient exerted an 
influence on the manifestation of the difference in therapeutic effect, and the therapeutic effect was more distinct in inpatients than in outpatients. It drew attention in relation to this finding that the difference in therapeutic effect was manifest in such a way that the therapeutic effect was more easily manifest in inpatients than in outpatients in the group given aluminum sucrose sulfate, whereas the therapeutic effect became manifest irrespective of inpatients or outpatients in the degraded carrageenin-given group. In other words, this may be considered that degraded carrageenin can be expected to be relatively effective even in outpatients in whom the therapeutic effect is not easily manifest.

The factorial items, which were of significance at a $5 \%$ or less level of significance in the way of manifestation of the therapeutic effect between degraded carrageenin and aluminum sucrose sulfate, were the fecal occult blood reaction, and the $x$-ray and endoscopic findings, viz., objective findings, respectively. Namely the difference in the way of manifestation of the therapeutic effect between the two agents was found to exist not in the subjective symptoms but in the objective findings. The fact that the $\mathrm{x}$-ray and endoscopic findings were ranked at the top, with high significance, having Radical 1 which represents the difference in therapeutic effect, at 0.430 ,** viz, at a $1 \%$ or less level of significance, indicates that both the difference in the therapeutic effect and the difference in the way of manifestation of the therapeutic effect were based on more objective factors. Examining Table 4 in detail, it is clear that the difference in the manifestation of therapeutic effect indicates superiority of degraded carrageenin.

It was noteworthy from the clinical point of view that the difference in therapeutic effect or the difference in the manifestation of therapeutic effect did not significantly vary with the locality of ulcer.

No side-effects worth particular mention were encountered throughout the entire study. Attention should be drawn, however, to the finding that a mild fall in the serum cholesterol level and a mild prolongation of the blood coagulation time occurred in one of the lactose cases. If these findings had been attained in cases given either degraded carrageenin or aluminum sucrose sulfate, the effect of the introduction of either agents into the blood stream through intestinal absorption cannot be denied, although it is known in general that antipepsin agents are hardly absorbed through the intestine when orally administered (Dohlman 1956; Dziewiatkowski 1956; Engel and Fahrenbach 1968; Ranney and Eich 1969). Accordingly, the lactose-given control group set up in the elinieal experiments may also be considered to be of significance in this sense.

\section{Acknowledgment}

Mr. T. Onoda is appreciated for his statistical assistance.

Degraded carrageenin was supplied from Nihon Chemipher Co., Tokyo, Japan, and aluminum sucrose sulfate was purchased from Chugai Pharmaceutical Co., Tokyo, Japan. 


\section{References}

1) Anderson, W. (1961) The efffect of a sulphated polysaccharide upon the diffusion of pepsin through mucin. $J$ : Pharmacol. exp. Ther., 13 (Suppl.), 122T-125T.

2) Anderson, W. \& Watt, J. (1959) The protective action of an algal polyanion against experimentally produced peptic uleeration in the guinea pig. J. Physiol., 147, 52-53.

3) Babkin, B.P. \& Komarov, S.A. (1932) The influence of gastric mucus on peptio digestion. Canxd, med, Ass. J., 27, 463-469.

4) Cook, D.L., Eich, S. \& Cammarata, P.S. (1963) Comparative pharmacology and chemistry of synthetic sulfated polysaccharides. Arch. int. Pharmacodyn., 144, 1-19.

5) Dohlman, C.H. (1956) The fate of the sulfate group of chondroitin sulfate after administration to rats. Acta physiol. scand., 37, 220-234.

6) Doll, R., Hill, I.D. \& Hutton, C.F. (1965) Treatment of gastric ulcer with carbenoxolone sodium and oestrogens. Gut, 6, 19-24.

7) Doll, R., Price, A.V., Pygott, F.\& Sanderson, P.H. (1956) Continuous intragastric milk drip in the treatment of uncomplicated gastric ulcer. Lancet, 1, 70-73.

8) Dziewiatkowski, D.D. (1956) Some aspects of the metabolism of chondroitin sulfate$\mathrm{S}^{35}$ in the rat. J. biol. Chem., 223, 239-249.

9) Engel, R.H. \& Fahrenbach, M.J. (1968) Intestinal absorption of heparin in the rat and gerbil (33422). Proc. Soc. exp. Biol. Med., 129, 772-777.

10) Horwich, L. \& Galloway, R. (1965) Treatment of gastric ulceration with carbenoxolone sodium: Clinical and radiological evaluation. Brit. med. J., 2, 1274-1277.

11) Houck, J.C., Bhayana, J. \& Lee, T. (1960) The inhibition of pepsin and peptic ulcers. Gastroenterology, 39, 196-200.

12) Ishimori, A. (1971) Mechanism of the antipeptic action of anionic carbohydrate and its elinical application for the treatment of peptic ulcer. Tohoku $J$. exp. Med., 1.03, 141-157.

13) Lawrence, J.S. (1952) Dietetic and other methods in the treatment of peptic ulcer. Lancet, 262, 482-485.

14) Levey, S. \& Sheinfeld, S. (1954) The inhibition of the proteolytic action of pepsin by sulfate-containing polysaccharides. Gastroenterology, 27, 625-628.

15) Martin, F., Vuez, J.L., Berard, A., Andrée, C. \& Lambert, R. (1968) A study of the mechanisms of inhibition of peptic proteolysis by a sulphated polysaccharide. Diges. tion, 1, 165-174.

16) Pipær, D.W. (1967) Antacid and anticholinergic drug therapy of peptic ulcer. Gastroenterology, 52, 1009-1018.

17) Ranney, R.E. \& Eich, S.J. (1969) The metabolism of sodium amylopectin sulfate in the rat. Toxicol. appl. Phamacol, 15, 593-603.

18) Sun, D.C.H. (1964) Long-term anticholinergie therapy for prevention of recurrences in duodenal ulcer. Amer. J. dig. Dis., 9, 706-716.

19) Zimmon, D.S., Miller, G., Cox, G. \& Tesler, M.A. (1964) Specific inhibition of gastric pepsin in the treatment of gastric ulcer. Gastroenterology, 56, 19-23. 